mixture at 7 torr. If the flow is insufficient, then the cooling system cannot remove enough heat to compensate for that which is being transferred into the tube through radiation and convection and no $\mathrm{CW}$ lasing occurs. However, at high flow rates the output power is large. At $310 \mathrm{~K}$ outlet temperature a CW output power greater than $8 \mathrm{~mW}$ was measured. As shown in Fig. 6, increasing the blackbody temperature increases the output power, as should be expected. The blackbody temperature determines the pumping power of this laser and also effectively controls the outlet temperature of the cooling gas when a constant flow rate is maintained. The results plotted in Fig. 6 were also obtained with the standard mixture and with a constant cooling gas flow. The linearity of the curve should be expected at the highest temperatures where the Rayleigh-Jeans approximation for the specific intensity of the radiation at the $4.3 \mu \mathrm{m}$ pumping band is accurate.

\section{CONCLUSIONS}

Experiments have been carried out which prove that pumping of gas lasers by the radiation field of a blackbody cavity is possible. Mixtures of $\mathrm{CO}_{2}, \mathrm{He}$, and $\mathrm{Ar}$ have been used and a parametric study has determined that the maximum output power is obtained with a 20 percent $\mathrm{CO}_{2}-15$ percent $\mathrm{He}-65$ percent Ar mixture. By appropriately varying the gas mixture, the blackbody temperature, and the flow rate of the cooling gas,
$\mathrm{CW}$ output powers of $8-10 \mathrm{~mW}$ have been achieved. More power may be obtained if other gases are added to the mixture.

\section{ACKNOWLEDGMENT}

The authors would like to thank Dr. R. J. DeYoung of NASA for his continued encouragement and support.

\section{REFERENCES}

[1] W. R. Weaver and J. H. Lee, "A solar simulator-pumped atomic iodine laser," Appl. Phys. Lett., vol. 39, no. 137, 1981.

[2] W: H. Christiansen, "A new concept for solar pumped lasers," in Progress in Aeronatics and Astronautics, vol. 16, Radiation Energy Conversion in Space, $\mathrm{K}$. Billman, Ed. New York: Amer. Inst. Aeronautics and Astronautics, 1978, pp. 346-356.

[3] P. A. Bokhan, "On optical pumping of a molecular laser by blackbody radiation," Opt. Spectrose, vol. 26, no. 423, 1969.

[4] H. Shirahata, S. Kawada, and T. Fujioka, "Atmospheric pressure cw $\mathrm{CO}_{2}$ laser pumped by a blackbody radiation," presented at the 5 th Conf. Chemical Molecular Lasers, St. Louis, MO, 1977.

[5] I. Wieder, "Flame pumping and infrared maser action in $\mathrm{CO}_{2}$," Phys. Lett., vol. 24A, no, 759, 1967.

[6] P. A. Bokhan, "Experiment on optical pumping of a carbon dioxide molecular laser," Opt. Spectrosc, vol. 32, no. 435, 1972.

[7] R. J. Insuik and W. H. Christiansen, "Blackbody-pumped $\mathrm{CO}_{2}$ laser experiment," ptesented at the AIAA 16th Fluid Plasma Dynamics Conf. Danvers, MA, 1983, AIAA Paper No. 83-1701.

[8] W. H. Christiansen and R. J. Insuik, "High Power Blackbody Pumped $\mathrm{CO}_{2}$ Lasers," in Proc. 4th Gas Flow Chemical Laser Conf., Stresa, Italy, 1982 .

\title{
CW Laser Action in Atomic Fluorine
}

\author{
J. J. ROCCA, J. D. MEYER, B. G. PIHLSTROM, AND G. J. COLLINS
}

\begin{abstract}
We have obtained $\mathrm{CW}$ laser action on four transitions in the doublet system of atomic fluorine for the first time. All previously reported laser action was on a pulsed basis only. CW laser radiation was obtained when $F_{2}$ or $A g F$ was used as a fiuorine donor in an electron beam pumped helium plasma. A multiline output power of $200 \mathrm{~mW}$ was obtained.
\end{abstract}

QEVERAL authors [1]-[6] have previously reported pulsed Slaser action in atomic fluorine. Kovacs and Uttree [1] obtained 1-2 $\mu$ s laser pulses on the $7037.5,7127.9$, and 7202.4 $\AA$ lines of atomic fluorine in either $\mathrm{CF}_{4}, \mathrm{SF}_{6}$, or $\mathrm{C}_{2} \mathrm{~F}_{6}$ and helium mixtures. The lower laser levels $\left(3 s^{2} P\right)$ are relatively depopulated via allowed resonant transitions and they con-

Manuscript received November 21, 1983; revised February 9, 1984. This work was supported by the National Science Foundation and is part of a joint project between Colorado State University and Spectra Physics, Mountain View, CA.

The authors are with the Department of Electrical Engineering, Colorado State University, Fort Collins, CO 80523 . cluded that radiation trapping of the lower level via reabsorption of the 955 and $956 \AA$ resonant radiation limited the laser pulse length. Jeffers and Wiswall [2] obtained quasi-CW laser pulses, $20 \% \mathrm{~s}$ long, in a He-HF discharge. They reported that the trapping of the resonant radiation was not a limiting factor in their experiment due to the existence of a second lower laser level de-excitation process involving the atomic $\mathrm{H}$ produced in the dissociation of HF via the reaction $\mathrm{F}\left(3 s^{2} P\right)+\mathrm{H}(n=1) \rightarrow$ $\mathrm{F}\left(2 p^{3}{ }^{2} P^{\circ}\right)+\mathrm{H}(n=5)+\Delta E$. However, their laser operated in the afterglow of the discharge pulse, precluding $\mathrm{CW}$ operation. More recently, Crane and Verdeyen [3] reported $80 \mu \mathrm{s}$ long laser pulses using a hollow cathode discharge. In this case the premature termination of the laser pulse with respect to the excitation pulse was suggested to be due to the depletion of the fluorine donor.

We report $C W$ laser action on the 7037.5, 7127.9, 7202.4, and $7800.2 \AA$ laser transitions of atomic fluorine using a $\mathrm{dc}$ electron beam to excite either $\mathrm{He}-\mathrm{F}_{2}$ or $\mathrm{He}-\mathrm{AgF}$ gas mixtures. The laser setup empolyed in this experiment was similar to the 


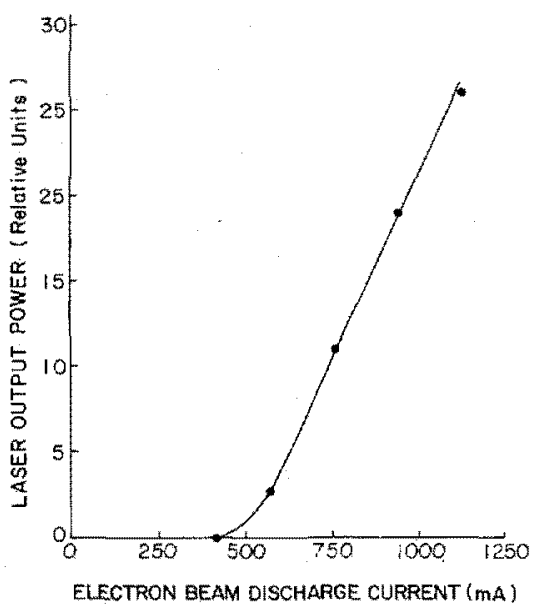

Fig, 1. Multiline laser output power of the red atomic fluorine laser transitions as a function of electron beam discharge current. He and $F_{2}$ partial pressures 1.9 torr and 35 mtorr, respectively. Axial magnetic field $3.2 \mathrm{kG}$

one we previously used to obtain $\mathrm{CW}$ laser action in several singly ionized species, and has been described in previous publications [7]. A multiline CW output power of $200 \mathrm{~mW}$ was obtained in a $\mathrm{He}-\mathrm{F}_{2}$ gas mixture at partial pressures of 1.9 torr and 35 mtorr, respectively. This maximum output power was obtained at an electron beam discharge current of $1.2 \mathrm{~A}$, an electron beam energy of $2 \mathrm{keV}$, and with an axial magnetic field of $3.2 \mathrm{kG}$. The magnetic field was used to help confine the electron beam in the active region. Fig. 1 shows the variation of the laser output power with electron beam discharge current in a $\mathrm{He}-\mathrm{F}_{2}$ mixture. $\mathrm{CW}$ laser action was also obtained using $\mathrm{AgF}$ as a fluorine donor, when a reservoir containing $\mathrm{AgF}$ connected to the plasma tube was heated above $500^{\circ} \mathrm{C}$. Laser action was obtained when helium was used as a buffer gas, as it was in all the cases in which pulsed laser action was previously reported $[1]-[6]$. When neon was used as a buffer gas, laser action was not obtained. However, the addition of only 50 mtorr of He to a 0.3 torr Ne buffer gas discharge resulted in $\mathrm{CW}$ fluorine laser oscillation. This indicates that helium plays an important role in the excitation mechanism of the upper laser levels.

Observation of the laser output with a $0.5 \mathrm{~m}$ spectrometer showed that oscillation can occur at two frequencies displaced with respect to the line center of the atomic spontaneous emission. Fig. 2 shows the spectrum of the $7127.9 \AA$ laser line for three partial pressures of $F_{2}$. This splitting of the laser lines is attributed to very different velocity distributions of the atomic fluorine in the upper $\left(3 p^{2} P\right)$ and lower $\left(3 s^{2} P\right)$ levels. The velocity distribution of the upper laser levels can be substantially broadened if these levels are excited by a process with an energy surplus, in which the energy difference is balanced by a gain in the kinetic energy of the atoms involved. The velocity distribution of the atoms in the lower laser levels is narrower as a result of the imprisonment of resonant radiation and subsequent lengthening of the effective lifetime. When the pressure of $F_{2}$ is reduced, the density of atomic fluorine in the ground state decreases and the trapping of the resonant radiation becomes less severe, increasing the gain at line center,

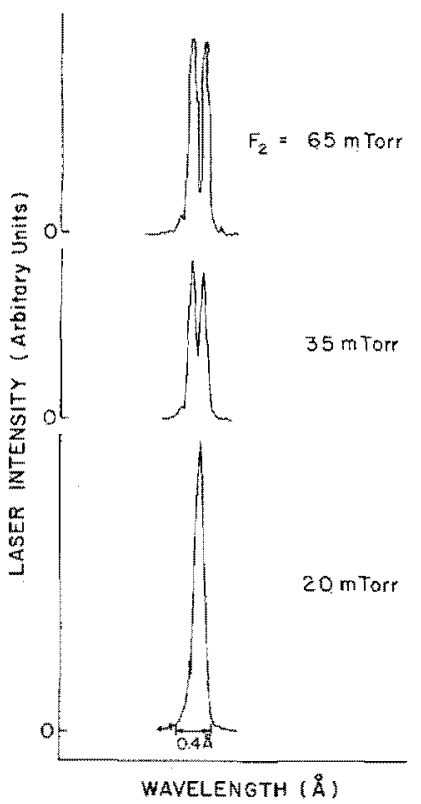

Fig. 2. Spectrum of the 7127.9 A laser output for three $F_{2}$ partial pressures. Helium balance to a total average pressure of 1.9 torr.

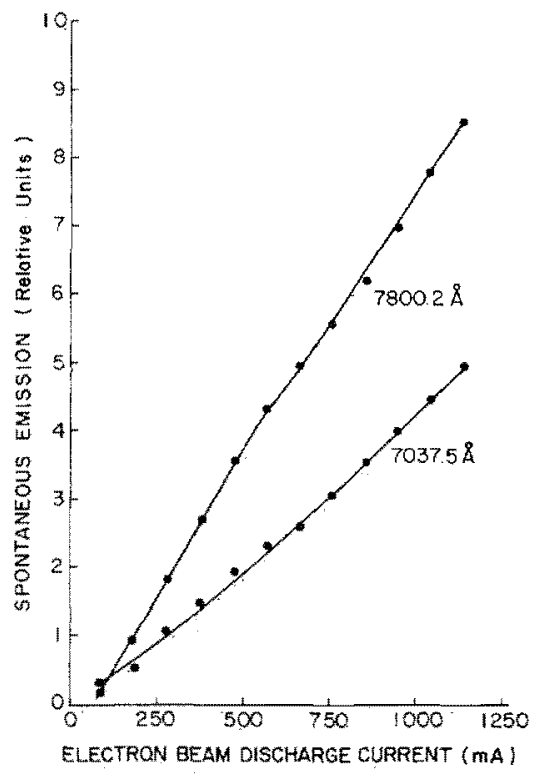

Fig. 3. Variation of the spontaneous emission of the 7037.5 and 7800.2 A atomic fluorine laser transitions as a function of electron beam discharge current. He and $F_{2}$ partial pressures 1.9 torr and 35 mtorr, respectively.

in agreement with Fig. 2. This characteristic of the spectral distribution of the laser light has been previously observed in the $8446 \AA \mathrm{Ar}-\mathrm{O}_{2}$ laser [8]-[10]. In this laser, the velocity distribution of the atoms in the $3 p^{3} P_{1,2}^{\circ}$ levels is broadened due to excitation involving collisions of the second kind between $\mathrm{O}_{2}$ and $\mathrm{Ar} *$ which have surplus energy. The gain profile is also split as a consequence of radiation trapping which occurs at line center on the strong resonant line at $1300 \AA$ connecting the lower laser level with $\mathrm{O} I$ ground state.

Fig. 3 shows the variation of the spontaneous emission of two laser transitions as a function of the electron beam discharge current. The spontaneous emission shows a linear 
dependence on the current as does the laser output power, indicating an excitation mechanism requiring a single electron collision. The dissociative excitation processes indicated in reactions (1) and (2), in which the helium metastable is assumed to be pumped by direct electron impact (3), are "single step" mechanisms

$$
\begin{aligned}
\mathrm{He}\left(2^{3} S\right)+\mathrm{F}_{2} & \rightarrow \mathrm{F}^{*}+\mathrm{F}+\mathrm{He}(1 s)+\Delta E \\
\mathrm{He}\left(2^{3} S\right)+\mathrm{AgF} & \rightarrow \mathrm{F}^{*}+\mathrm{Ag}+\mathrm{He}(1 s)+\Delta E \\
\mathrm{e}^{-}+\mathrm{He}(1 s) & \rightarrow \mathrm{He}\left(2^{3} S\right)+\mathrm{e}^{-} .
\end{aligned}
$$

Reactions (1) and (2) have a surplus energy $\Delta E$ of 3.7 and $1.6 \mathrm{eV}$, respectively. Notice that in dissociative excitation a close energetic resonance discrepancy is not required for efficient transfer [11]. For example, the energy discrepancy in the dissociative collision of $\mathrm{Ar}^{*}$ and $\mathrm{O}_{2}$ in the atomic oxygen laser operating at $8446 \AA$ is $2 \mathrm{eV}$ [12], [13]. This excess energy must, however, be carried off as kinetic energy by the products of the reaction. This super thermal velocity distribution is consistent with the split line profile of the laser lines observed experimentally. The helium excited state is indicated as the $2^{3} \mathrm{~S}$ state because this is the most abundantly populated level in an electron beam excited negative glow helium discharge [14]. The $\operatorname{He}\left(2^{1} S\right)$ metastable is efficiently destroyed by superelastic collisions with electrons and is converted to the $\mathrm{He}\left(2^{3} S\right)$ metastable. However, this and other excited states of helium can also contribute to (1) and (2). Notice that the metastable atoms of Ne do not have enough energy to satisfy (2). This also consistent with our experimental observation that no laser action is obtained with $\mathrm{Ne}$ as a buffer gas.

Another possible excitation mechanism involving helium metastable levels is

$$
\mathrm{He}\left(2^{3} S\right)+\mathrm{F}_{2} \rightarrow \mathrm{F}^{+}+\mathrm{F}+\mathrm{He}(1 s)+\mathrm{e}^{-}
$$

followed by three-body electron recombination

$$
\mathrm{F}^{+}+2 \mathrm{e}^{-} \rightarrow \mathrm{F}^{*}+\mathrm{e}^{-} \text {. }
$$

This mechanism, however, would not cause a difference in the velocity distribution of the laser levels since in (4) most of the excess energy would be taken by the resulting electron.

Other possible mechanisms exciting the upper laser levels are two-body ion-ion recombination reactions such as

$$
\begin{aligned}
& \mathrm{He}^{+}+\mathrm{F}^{-} \rightarrow \mathrm{F}^{*}+\mathrm{He}+\Delta E \\
& \mathrm{He}^{+}+\mathrm{F}^{-} \rightarrow \mathrm{F}^{+}+\mathrm{He}+\mathrm{e}^{-}+\Delta E \\
& \mathrm{He}^{+}+\mathrm{F}_{2}^{-} \rightarrow \mathrm{F}^{+}+\mathrm{He}+\mathrm{e}^{-}+\Delta E
\end{aligned}
$$

where (7) and (8) are followed by three-body electron recombination. Miller and Morgner [11] predict large cross sections for (6) and (7). Notice, however, that this process requires at least two electrons to create the reactant ions since one electron is required in the dissociative attachment (or attachment) process and another in ionizing He. Consequently, it is not consistent with our observation of a linear increase in the spontaneous emission with current. Reactions of negative fluorine ions with Ne ions would also be allowed from an energetic point of view.
Reaction (1) seems to be the most likely excitation mechanism of the $\mathrm{He}-\mathrm{F}_{2}$ laser. The production rate of metastable atoms in our electron beam discharge has been calculated to be sufficient to provide the needed pumping rate, through (1), for the measured $0.2 \mathrm{~W}$ laser output power. However, when Ar was added to the plasma with a 2 torr $\mathrm{He}-\mathrm{F}_{2}$ mix ture in an $\mathrm{Ar}: \mathrm{F}_{2}$ proportion of up to $4: 1$, with the purpose of quenching the helium metastables, the electron beam current necessary to achieve the threshold for laser action increased by only 20 percent. This is not sufficient, however, to rule out (1) as the major excitation mechanism since the cross section for quenching helium metastables by $F_{2}$, which is not available in the literature, could be several times larger than the one corresponding to $\mathrm{He}^{*}$-Ar collisions, which is $5.3 \AA^{2}$ [15]. For example, the cross section for quenching $\mathrm{He}\left(2^{3} S\right)$ by $\mathrm{SF}_{6}$ was measured to be $20.6 \AA^{2}$ [15]. Clearly, a more detailed study is necessary to determine the dominant excitation mechanisms of the $\mathrm{CW}$ electron beam pumped fluorine laser.

Finally, we observed CW laser action on the $8446 \AA$ line of atomic oxygen exciting a $\mathrm{He}-\mathrm{O}_{2}$ mixture with a dc electron beam in the same experimental setup. CW laser action was also obtained when the electron beam was used to excite pure oxygen gas. The output power on this transition was, however, only $1 \mathrm{~mW}$ using totally reflecting mirrors.

Summarizing, we have obtained $\mathrm{CW}$ laser action on the red lines of atomic fluorine for the first time. This was obtained using $\mathrm{F}_{2}$ or $\mathrm{AgF}$ as a fluorine donor in an electron beam excited helium plasma. A collisional excitation reaction with an energy surplus populates the upper laser level, causing a difference in the velocity distribution of the atoms in the upper and lower laser levels. This avoids the self-termination of the laser output caused by trapping of the lower state resonant radiation observed in previous studies. Direct current electron beams are thereby demonstrated to be a suitable method to excite $\mathrm{CW}$ atomic lasers.

\section{REFERENCES}

[1] M. A. Kovacs and C. J. Ultee "Visible laser action in fluorine I" Appl. Phys. Lett., vol. 17, pp. 39-40, 1970.

[2] W. Q. Jeffers and C. E. Wiswatl, "Laser action in atomic fluorine based on collisional dissociation of HF," Appl. Phys. Lett., vol. 17, pp. 444-447, 1970.

[3] J. K. Crane and J. T. Verdeyen, "The hollow cathode helium fluorine laser," J. Appl. Phys., vol. 51, pp. 123-129, 1980.

[4] I. J. Bigio and R. F. Begley, "High-power visible laser action in neutral atomic fluorine," Appl. Phys. Lett., vol. 28, pp. 263-264, 1976.

[5] T. R. Loree and R. C. Sze, "The atomic fluorine laser: Spectral pressure dependence," Opt. Commun., vol. 21, pp. 255-257, 1977.

[6] J. E. Lawler, J. W. Parker, L. W. Anderson, and W. A. Fitzsimmons, "Experimental investigation of the atamic fluorine laser," IEEE J. Quantum Electron., vol. QE-15, pp. 609-613, 1979.

[7] J. J. Rocca, J. D. Meyer, and G. J. Collins, "1-W $\mathrm{Zn}$ ion laser," Appl. Phys. Lett., vol. 43, pp. 37-39, 1983; see also and J. J. Rocca, J. D. Meyer, G. Fetzer, Z. Yu, and G. J. Collins, Conf. Lasers Electro-Optics, Baltimore, MD, May 1983.

[8] S. G. Rautian and P. L. Rubin, "On some features of gas lasers containing mixtures of oxygen and rare gas," Opt. Spectrosc., vol. 18 , pp. $180-181,1965$.

[9] L. N. Tunitskii and E. M. Cherkasov, "Pulse mode generation in an argon-oxygen laser," Opt. Spectrosc., vol. 26, pp. 344-346, 1969. 
[10] - "The mechanism of laser action in oxygen-inert gas mix tures," Opt. Spectrosc., vol. 18, pp. 154-157, 1967.

[11] W. A. Miller and H. Morgner, "A unified treatment in penning ionization and excitation transfer," J. Chem. Phys, vol. 67, pp. $4923-4930,1977$.

[12] C. Wilet, Introduction to Gas Lasers: Population Inversion Mechanisms. Oxford, England: Pergamon, 1974.

[13] W. R. Bennett, W. L. Faust, R. A. McFarlane, and C. K. N. Patel, "Dissociative excitation transfer and optical faser oscillation in
$\mathrm{Ne}-\mathrm{O}_{2}$ and $\mathrm{Ar}-\mathrm{O}_{2}$ if discharges," Phys. Rev. Lett., vol. 8, pp. $470-473,1962$.

[14] J. J. Rocca, "Electron beam pumping of cw ion lasers," Ph.D. dissertation, Colorado State University, Fort Collins, CO, 1983; see also J. R. McNeil, "New sputtered metal vapor laser systems," Ph.D. dissertation, Colorado State University, Fort Collins, CO, 1977.

[15] A. L. Schmeltekopf and F. C. Fehsenfeld, "De-excitation rate constants for helium metastable atoms with several atoms and molecules," J. Chem. Phys, vol. 53, pp. 3173-3177, 1970.

\title{
Active-Passive Mode Locking of an Nd:Phosphate Glass Laser Using \#5 Saturable Dye
}

\author{
LAWRENCE S. GOLDBERG AND PAUL E. SCHOEN
}

\begin{abstract}
An acoustooptic loss modulator and Eastman $\$ 5$ saturable absorbing dye are used to stably mode lock a TEM 00 Nd:phosphate glass oscillator. This hybrid approach has enabled reliable generation of $\sim 6$ ps duration pulses largely free of occurrences of satellite pulse structure.
\end{abstract}

$\mathrm{T}$ HE stability of passively mode-locked $\mathrm{Nd}$ lasers has been improved greatly in recent years, principally through use of intracavity acoustooptic loss modulators [1]-[6]. In this hybrid approach, the active loss modulation reduces the inherent statistical nature of the early stage pulse buildup, while retaining the effect of the saturable absorber as nonlinear loss element and $Q$-switch for final stage pulse shortening. For Nd:YAG lasers [3]-[6], active-passive mode locking has produced pulses essentially as short as those obtained by purely passive mode locking $(\sim 30 \mathrm{ps})$, but with important added advantages of high amplitude and pulse width stability, absence of satellite pulses, high peak-to-background contrast, sharp lasing threshold, and no missed shots. While many of these features have also been reported for $\mathrm{Nd}$ :glass lasers [1]-[3], considerably less data are available on their behavior in the very short pulse regime $(\sim 6 \mathrm{ps})$. Tomov et al. [2] used electrooptic modulation in a ring cavity laser and reported reliable mode locking and generation of transform-limited pulses down to $15 \mathrm{ps}$ duration. Seka and Bunkenburg [3] obtained minimum pulse widths of $\geqslant 25$ ps in studies of a phosphate glass oscillator containing bandwidth-limiting etalons. They also observed occasional random satellite pulses for operation at pulse durations $<80 \mathrm{ps}$.

In this letter, we report studies of active-passive mode locking of a Nd: phosphate glass laser using Eastman dye $\$ 9860$ and

Manuscript received December 5, 1983.

The authors are with the Naval Research Laboratory, Optical Sciences Division, Washington, DC 20375. the new chemically more stable heptamethine pyrylium dye \#5 [7], [8] as saturable absorbers. Dye $\$ 9860$ (and $\$ 9740$ ) has generally been used as a fast relaxing $(\tau \sim 7 \mathrm{ps})$ saturable absorber for passive mode locking of Nd lasers. Dye \#5 was found to have a substantially shorter relaxation time $(\tau=2.7 \mathrm{ps})[8]$, but as a consequence saturates at a significantly higher flux than does \#9860. Alfano et al. [9] recently reported passive mode locking and short pulse generation with dye $\# 5$ in a multitransverse mode glass laser. However, Kolmeder and Zinth [10] found that because of its higher saturation flux they could not obtain passive mode locking with dye \#5 when substituted directly into a contact dye cell in a standard TEM Oo $_{0}$ cavity. By using a folded-cavity geometry to provide a focused higher. intensity region for the dye, they were then able to obtain very good mode-locking behavior and generated pulses of a few picoseconds duration. We have confirmed this latter behavior of dye \#5 in our laser by attempting purely passive mode locking with the RF power to the acoustooptic modulator turned off. Even at fairly high pumping levels and high dye concentrations only conventional lasing was observed, whereas \#9860 would readily saturate and passively mode lock. However, with use of the acoustooptic modulator we have been able to demonstrate excellent mode locking using dye \#5. In addition to generating more stable and shorter pulses than with $\# 9860$ in an active-passive system, use of dye $\# 5$ has also sharply reduced the tendency toward producing satellite pulses.

In our experiments, an Intra Action ML-57C acoustooptic modulator operating at $57 \mathrm{MHz}$ RF (114 MHz optical) was incorporated into a passively mode-locked oscillator cavity described elsewhere [11]. The modulator was placed adjacent to the flat output mirror $(R=55$ percent), which was mounted on a precision translation stage. It was operated in pulsed mode, to minimize thermal loading, at a maximum RF power of $10 \mathrm{~W}$. Water cooling temperature was controlled to $\pm 0.2^{\circ} \mathrm{C}$ to ensure long-term stability, RF drive was derived from a frequency 